\title{
Proper motions of water masers: W 51 North, Main, and South
}

\author{
Hiroshi Imai, Tetsuo Sasao, Osamu Kameya \\ VERA Project Office, NAOJ, Mitaka, Tokyo 181-8588, Japan
}

Teruhiko Watanabe, Toshihiro Omodaka, Masanori Nishio

Dept. of Physics, Kagoshima University, Kagoshima 890-0065, Japan

\author{
Yoshiharu Asaki \\ Institute Space and Astronautical Science, Sagamihara, Kanagawa \\ 229-0022, Japan
}

Jun-ichi Nakajima

KSRC, Communications Research Laboratory, Kashima, Ibaraki 314-0012, Japan

\begin{abstract}
We describe the proper motion measurements of water masers in the massive-star forming region $\mathrm{W} 51 \mathrm{~A}$ and the analysis of the 3-D kinematics in three maser clusters of $\mathrm{W} 51 \mathrm{~A}$. We found a systematic expanding motion in one of the clusters named W 51 North, while no systematic motion was detected in other two clusters named W 51 Main and South. The 3-D motions of the clusters relative to the position reference feature in W 51 North indicate a separating motion between the W 51 North and the W 51 Main/South clusters. We estimated the distance to W 51 North as to be $6.7 \pm 2.1 \mathrm{kpc}$ on basis of the statistical parallax and the model fitting methods.
\end{abstract}

\section{Introduction}

Elucidating internal motions of giant molecular clouds (GMCs) is a key item to understand formation mechanism of massive-star clusters. As star formation mechanisms, a step-by-step process caused by the propagation of shock layers from formed HII regions, or cloud contraction by the self-gravitation due to a huge GMC's mass has been considered. The bulk motions among adjacent star-forming regions in a GMC should trace such processes. VLBI monitoring observations of water masers in the $\mathrm{W} 51 \mathrm{~A}$ region provides one of the best opportunities to measure the 3-D motions among the star-forming regions (maser clusters), W 51 North, Main, and South that are located within $70^{\prime \prime}$.

\section{Observations and Data Reduction}

The VLBI observations of water masers in W $51 \mathrm{~A}$ were made five times, on February 21, April 2, May 6, June 6, and October 21 in 1999, with the Japanese 

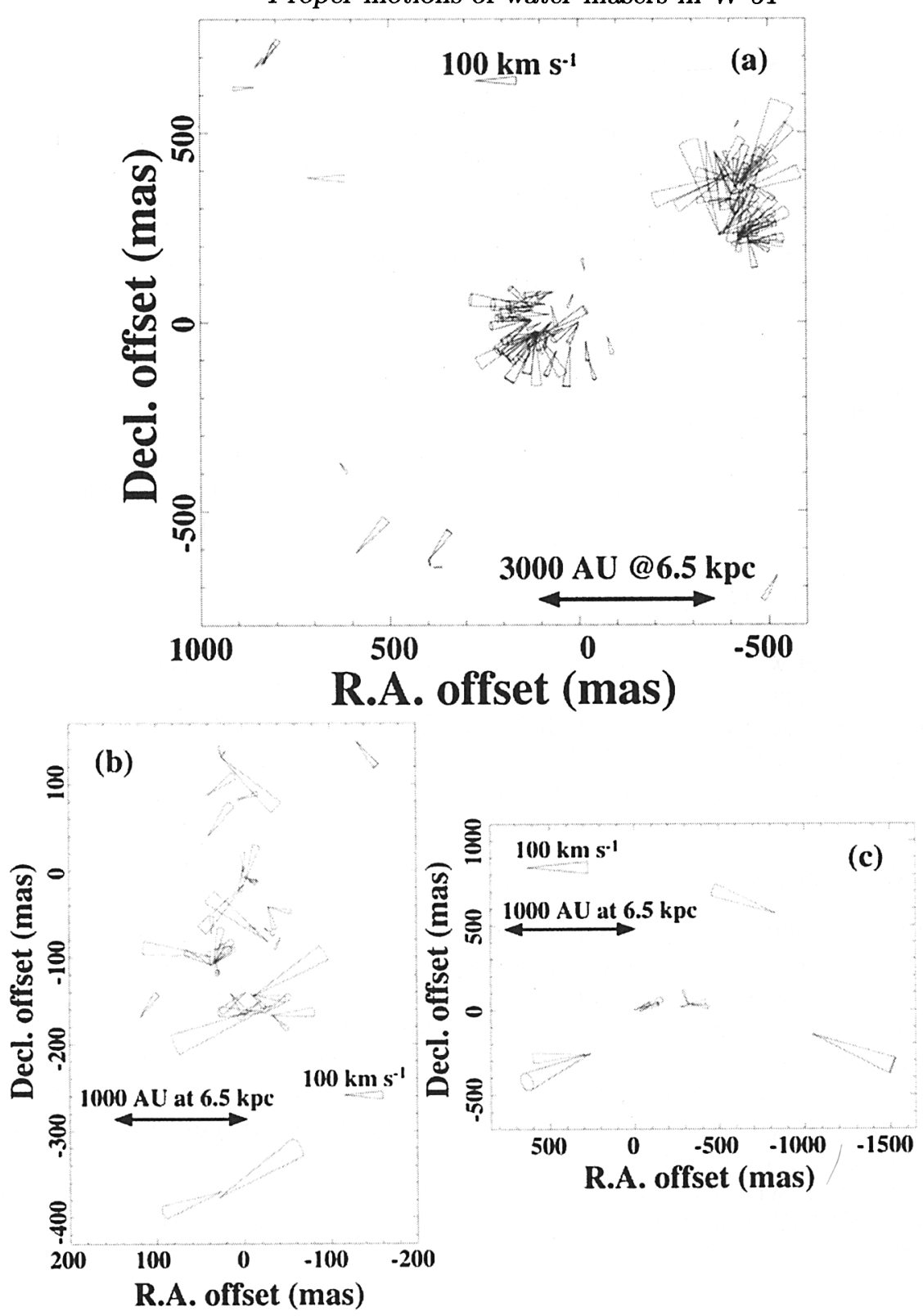

Figure 1. Proper motions of water maser features in the $\mathrm{W} 51 \mathrm{~A}$ region. A position, opening direction, and length of a cone indicate the position of a maser feature, and the direction and amplitude of its proper motion, respectively. An inclination of a maser motion is calculated with respect to the systemic radial velocity of the parent molecular cloud. (a) in W 51 North; (b) in W 51 Main (near the HII region e1, Gaume et al. 1993); (c) in W 51 South (near the HII region e8). An origin of each map is located at the position-reference maser feature in each region. 
domestic VLBI network, J-Net. The narrowest beam size of the element antennas at $22 \mathrm{GHz}$ is $72^{\prime \prime}$, and covers the three water maser clusters. Thus, these clusters were observed simultaneously. The received data were recorded with the VSOP terminal in one base band channel with a band width of $16 \mathrm{MHz}$ that corresponds to a velocity coverage of $220 \mathrm{~km} \mathrm{~s}^{-1}$. The data correlation was made with the Mitaka FX correlator. The correlation process was made three times by shifting the phase-tracking centers to the locations of these three clusters.

Data reduction was made with the AIPS package. Fringe fitting and selfcalibration procedures were made for a bright maser spot in W 51 North. The solutions were applied to all of the data, then maser maps were made for these clusters. The synthesized beam size in the five observations was typically 3 mas. The relative position accuracy of a maser feature was ranged in 0.05-0.5 mas depending on a signal-to-noise ratio and a spatial structure of the feature.

\section{Results and Discussion}

The numbers of water maser features identified at least in two observing epochs were 123, 48, and 10 in W 51 North, Main, and South, respectively. These numbers are larger than those of the previous observations for W 51 North by Schneps et al.(1981) and for W 51 Main by Genzel et al.(1981). Figure 1 shows relative proper motions of water masers in W $51 \mathrm{~A}$ in the present work.

\subsection{W 51 North}

We found a systematic expansion motion with a clear bipolarity in W 51 North. Fitting the 3-D motions of maser features to a radially-expanding flow model, we estimated at first the bulk motion and the position of the flow origin. This procedure is almost the same as that by Gwinn et al.(1992). These parameters are important ones for the relative 3-D motions in the GMC of W 51A.

Then we estimated the distance to W 51 North as to be $6.3 \pm 1.2 \mathrm{kpc}$ and $7.2 \pm 1.7 \mathrm{kpc}$ by the above model-fitting and the statistical parallax methods, respectively. The averaged value of the distance obtained from the two methods is $6.7 \pm 2.1 \mathrm{kpc}$, which is consistent with that obtained by the previous measurement $(8.3 \pm 2.5 \mathrm{kpc}$, Schneps et al. 1981) and the kinematic distance, $\sim 5.5$ $\mathrm{kpc}$.

\subsection{W 51 Main and South}

In W 51 Main, we did not find any systematic motion in the maser features. Our result is consistent with that of Genzel et al.(1981). Both of the results suggest that the kinematics in W 51 Main is fundamentally random. However, Leppanën et al.(1998) reported a systematic stream motion in the small velocity coverage of $\mathrm{V}_{L S R}=54-68 \mathrm{~km} \mathrm{~s}^{-1}$. It is likely that such a stream motion is created only at this velocity coverage that is almost equal to the systemic velocity of the adjacent cloud. There may exist an interaction between the random flow and the cloud with a systematic motion (Leppanën et al. 1998). 


\begin{tabular}{rccccl}
\hline \hline & $\mathrm{V}_{X}$ & $\mathrm{~V}_{Y}$ & $\mathrm{~V}_{Z}\left(\mathrm{~V}_{L S R}\right)$ & Features \\
\hline W 51 North (average) & $\ldots \ldots \ldots$ & $-59 \pm 23$ & $43 \pm 39$ & $60 \pm 35$ & 121 \\
(model fit) $\ldots \ldots \ldots \ldots$ & $-51 \pm 16$ & $12 \pm 22$ & $59 \pm 16$ & 83 \\
W 51 Main (e2) (average) $\ldots \ldots$ & $66 \pm 50$ & $99 \pm 23$ & $81 \pm 29$ & 37 \\
W 51 South (e8) (average) & $\ldots$ & $-26 \pm 72$ & $62 \pm 27$ & $71 \pm 30$ & 10 \\
\hline
\end{tabular}

Table 1. Relative bulk motions of three maser clusters with respect to the position-reference feature in W 51 North.

We revealed a bipolar flow in W 51 South with an expansion velocity up to $100 \mathrm{~km} \mathrm{~s}^{-1}$. However, it is still a marginal level due to the small number of the measured proper motions.

\subsection{Relative 3-D motions among the water maser clusters}

Because we measured the positions of all water maser features with respect to the position-reference feature in W 51 North, we could also measure the 3-D relative systemic velocities of the individual maser clusters, or the $3-\mathrm{D}$ relative motions among the star-forming regions W 51 North, Main, and South. Only for W 51 North, the systemic velocity could be also estimated from the velocity of the flow origin, which was derived from the model fitting procedure.

Table 1 gives the obtained systemic velocities of the water maser clusters. The uncertainty of these values are large (several tens of $\mathrm{km} \mathrm{s}^{-1}$ ) when the uncertainty is defined by the standard deviation of the maser motions. Even in the model fitting procedure, the uncertainty is large due to large deviations of the maser kinematics from the modeled expanding flow. Nevertheless, a large separating motion is seen between W 51 North and Main over the uncertainty. There are two possibilities to explain this motion. The first is due to a true separating motion driven by pressures by the adjacent HII regions. In fact, there are bright HII regions at the west of W 51 Main/South and at the north-west of W 51 North. However, such a fast motion prefers other input sources. The second is due to bias in the obtained average systemic velocities of the clusters. In fact, the radial velocities in W 51 Main are biased to a motion receding from us with respect to the systemic velocity of the parent molecular cloud. Thus, a caution should be paid for estimation of bulk motions of maser clusters.

Acknowledgments. We acknowledge all staff members and students who have helped in array operation and in data correlation in J-Net . H. I. was financially supported by the Research Fellowship of the Japan Society of the Promotion of Science for Young Scientist.

\section{References}

Gaume, R.A., Johnston, K.J., \& Wilson, T.L. 1993, ApJ, 417, 645

Genzel, R., et al. 1981, ApJ, 248, 1039

Gwinn, C.R., Moran, J.M., \& Reid, M.J. 1992, ApJ, 393, 149

Leppänen, K., Liljeström, T., \& Diamond, P.J. 1998, ApJ, 507, 1998

Schneps, M.H., et al. 1981, ApJ, 249, 124 